\title{
Methodologie und methodisches Vorgehen
}

\subsection{Fragestellung und Auswahl der Studiengänge}

In der vorliegenden Untersuchung wurde auf Grundlage der dargestellten theoretischen Verknüpfungen der Frage nachgegangen, wie sich Studien(fach)wahlen im Kontext von sozialem Milieu und Geschlecht konstituieren. Um dieser Frage nachzugehen, wurde ein qualitativer Zugang gewählt: Im Gegensatz zu quantitativer Forschung wurden keine vordefinierten Hypothesen an den Forschungsgegenstand herangetragen, die überprüft werden sollten. Stattdessen bietet sich ein qualitatives Vorgehen an, um theoriegeleitet offene Fragestellungen zu verfolgen und das beforschte Feld möglichst umfassend zu, verstehen' (Krüger 2000: 324).

Dazu wurden Studierende im ersten Semester einer Hochschule für Angewandte Wissenschaften (ehemals ,Fachhochschule ${ }^{11}$ ) aus zwei unterschiedlichen Fachbereichen bzw. Studiengängen befragt. Dieser frühe Zeitpunkt bietet sich an, da die Entscheidung für das Studienfach erst kurz zurück liegt und die Ausbildung eines Fachhabitus noch nicht weit fortgeschritten sein kann. Die Auswahl der Fächer erfolgte aufgrund verschiedener theoriegeleiteter Vorannahmen: Mit Rückgriff auf das zahlenmäßige Geschlechterverhältnis (s. Abschnitt 2.1), die vergeschlechtlichte Professionalisierung der entsprechenden

\footnotetext{
${ }^{1}$ Die Wahl gerade dieses Hochschultyps bietet sich auch deshalb an, weil er in Untersuchungen zu Passungsverhältnissen im Bildungssystem häufig vernachlässigt wird (Evertz/Schmitt 2016: 163)
}

Elektronisches Zusatzmaterial Die elektronische Version dieses Kapitels enthält Zusatzmaterial, das berechtigten Benutzern zur Verfügung steht https://doi.org/10.1007/978-3-658-32445-2_5 
Berufsfelder (s. Abschnitt 3.1.6) und die Erkenntnisse der Fachkulturforschung (s. Abschnitt 3.3.2.4) wurden zwei möglichst kontrastive Studiengänge ausgewählt:

- Bauingenieurwesen (angestrebter Abschluss: Bachelor of Science)

- Soziale Arbeit (angestrebter Abschluss: Bachelor of Arts)

Zugleich konnte eine Gemeinsamkeit der beiden Studierendenschaften dahingehend erwartet werden, dass sie jeweils einen relativ heterogenen Bildungshintergrund haben: So gelten sowohl die Ingenieurwissenschaften wie auch die Sozialwissenschaften an Hochschulen für Angewandte Wissenschaften als Studienbereiche, in denen sich vergleichsweise viele ,Bildungsaufsteiger*innen “ finden - in denen die akademische Reproduktion also nicht so hoch ist wie etwa in der universitären Medizin. Gleichzeitig war zu vermuten, dass die Studierenden der beiden Fächer im sozialen Raum nach Bourdieu schwerpunktmäßig in unterschiedlichen Bereichen verortet werden können: So weisen die Erkenntnisse von Bourdieu und der anknüpfenden Milieuforschung nicht nur darauf hin, dass es eine Homologie zwischen den Soziallagen und Berufen der Menschen und ihrem Habitus gibt (Lange-Vester/Teiwes-Kügler 2013a: 154), sondern auch, dass Studierende je nach Disposition eine Affinität zu bestimmten Fächern haben (Lange-Vester/Teiwes-Kügler 2004). Es kann davon ausgegangen werden, dass auch jedes Milieu ,typische Schwerpunkte“ (Vester 2001: 226) im Raum der beruflichen Positionen hat, die sich nach der bevorzugten Art und Kombination von kulturellem und ökonomischem Kapital richtet und dass Sozialarbeiter*innen hier stärker zum kulturellen Pol, Bauingenieur*innen dagegen stärker zum ökonomischen Pol hin tendieren (ebd.: 416 ff.). Zudem kann bei Bauingenieur*innen ein höheres Gesamtvolumen an Kapital vermutet werden und damit eine höhere vertikale Positionierung im Raum der beruflichen Positionen (ebd.). ${ }^{2}$ Auch wenn sich die Studierenden (noch) nicht im Beruf befinden, sondern in der letzten Qualifizierungsphase dorthin, lag die Vorannahme nahe, dass man es bei den Studierenden der Sozialen Arbeit und des Bauingenieurwesens an einer Hochschule für Angewandte Wissenschaften mit unterschiedlichen schwerpunktmäßigen habitus- und milieuspezifischen Musternund auch entsprechend unterschiedlich vergeschlechtlichen Habitusmustern - zu tun hat. Noch dazu konnte davon ausgegangen werden, dass auch innerhalb eines Fachs eine Vielfalt an habitus- und milieuspezifischen Hintergründen $\mathrm{zu}$ finden ist (Lange-Vester/Teiwes-Kügler 2004). Von einer homogenen Studierendenschaft

\footnotetext{
${ }^{2}$ Was keine kausale Aussage über die Milieuverortung der Studierenden bzw. Bauingenieur*innen zulässt.
} 
konnte demnach wiederum nicht ausgegangen werden. Es wurde vorab also eine gewisse habitusbezogene Fachhomogenität bei gleichzeitiger intrafachlicher Varianz vermutet.

Beide Studienfächer wurden auch vor dem Hintergrund ausgewählt, dass in der aktuellen Forschung zwar die Wege von Frauen in die Ingenieurfächer umfassend rekonstruiert werden - auch regelmäßig die von Männern als zukünftige Ingenieure -, aber nur teilweise jene von Männern in die Soziale Arbeit. Die Wege von Frauen in die Soziale Arbeit waren in der Forschung bisher von noch geringerem Interesse. Wie ebendiese Passung entstehen kann jenseits von binären Zuschreibungen vermeintlich spezifisch-weiblicher , sozialer Kompetenzen", sondern vor dem Hintergrund einer differenzierten Habitusanalyse, ist daher besonders interessant.

Die gegensätzlichen Geschlechterverhältnisse in den untersuchten Fächern (jeweils 70-75 \% zu 30-25\%), die bisherigen Erkenntnisse der Geschlechterforschung $\mathrm{zu}$ Studien(fach)wahlen in technischen und sozialen Fächern (s. Abschnitt 3.1) und die vergleichsweise privilegierte berufliche Position von Bauingenieur*innen gegenüber Sozialarbeiter*innen deuten außerdem auf ein spezifisches Zusammenwirken der Dimensionen von Klasse bzw. Milieu und Geschlecht hin, das zur strukturellen Abdrängung von Frauen aus dem Bauingenieurwesen und zu einer geringeren kulturellen Passung von Männern zur Sozialen Arbeit führt. Gleichzeitig war eine Nähe von ,bestimmten`Frauen zum Bauingenieurwesen und ,bestimmten“ Männern zur Sozialen Arbeit zu vermuten ${ }^{3}$. Wie diese Nähe und die Passung zur jeweiligen Fachkultur vor dem Hintergrund des vergeschlechtlichten und vergeschlechtlichenden Milieuhabitus der Befragten zustande kommt, ist die leitende Frage, die in der Untersuchung verfolgt wurde.

\subsection{Feldzugang und Auswahl der Interviewpersonen}

Unter Abwägung von Machbarkeit und Aussagekraft wurde geplant, insgesamt zwölf Interviews zu führen mit je drei Studentinnen sowie drei Studenten aus den beiden Studienfächern. Um Studierende für die Interviews zu gewinnen, wurde Kontakt mit Professor*innen der beiden Fachbereiche aufgenommen, um in ausgewählten Seminaren die Studierenden persönlich anzusprechen. So sollte eine Vorauswahl durch die Lehrenden vermieden werden. In den zwei besuchten Seminaren wurde den Studierenden das Forschungsvorhaben vorgestellt und transparent kommuniziert, dass es um die Frage geht, wie Studien(fach)wahlen

\footnotetext{
${ }^{3}$ Bezogen auf spezifische Habitusmuster.
} 
zustande kommen. Es wurde betont, dass eine Teilnahme an den Interviews sowohl für die Forschungsergebnisse sehr wichtig ist als auch einen Raum bietet für die persönliche Reflexion der eigenen Studienentscheidung. Außerdem wurde verdeutlicht, dass die Studierenden in den Interviews über ihre eigenen Vorstellungen des Studiums und ihre persönlichen Erfahrungen mit ihrer Studienentscheidung erzählen sollten und somit keine, Vorkenntnisse" nötig seien oder weitere Voraussetzungen erfüllt sein müssten. Und schließlich wurden die Studierenden vorab mündlich über die Anonymisierung der Interviews aufgeklärt. Nach einem anschließenden Austausch mit den Studierenden sowie ersten Interessensbekundungen haben sich letztendlich insgesamt 51 Studierende für die Teilnahme an einem Interview bereit erklärt und ihre Emailadresse für die Terminabsprache hinterlassen.

Der überwiegende Anteil (39 Studierende) stammte dabei aus der Sozialen Arbeit (davon 32 weiblich). Das kann zum einen auf die inhaltliche Nähe der Studierenden zu sozialwissenschaftlichen Forschungsmethoden zurückzuführen sein - die Teil ihres Modulplans sind und möglicherweise ihre Neugier wecken und zum anderen auf die Nähe zur Forscherin, welche die Studierenden zwar nicht persönlich kannten, die aber selbst einen Abschluss in der Sozialen Arbeit hat und sich entsprechend vorstellte.

Unter den zwölf Studierenden des Bauingenieurwesens, die sich für ein Interview gemeldet hatten, waren acht weiblich, was angesichts des geringen Anteils an Frauen in diesem Studiengang selbst (ca. $25 \%$ ) verhältnismäßig viele weibliche Interessentinnen sind. Dies spricht dafür, dass nicht nur eine gewisse fachkulturelle Nähe bzw. Distanz die Interviewbereitschaft beeinflusst, sondern auch das Geschlecht eine Rolle gespielt hat. Offenbar war die Distanz bzw. Skepsis gegenüber der sozialwissenschaftlichen Untersuchung und der Forscherin unter den männlichen Bauingenieurstudenten am stärksten ausgeprägt.

Um aus der großen Anzahl an Interessent*innen der Sozialen Arbeit eine handhabbare Anzahl an möglichst kontrastiven Fällen herauszufiltern, wurde im nächsten Schritt eine kurze Online-Befragung unter den Studierenden durchgeführt, um einige Basisinformationen zu erheben. Dazu gehörten unter anderem deren Bildungsabschluss, ihre Wohnsituation, etwaige Berufsabschlüsse, ihre Selbstzuordnung zu einem Geschlecht sowie ihre grundsätzliche Vorstellung vom Studium. Anschließend wurden sieben Studierende der Sozialen Arbeit zu einem Interview eingeladen. Entscheidend für diese Auswahl an Interviewpartner*innen waren mehrere Faktoren: Zum einen wurde ein etwa ausgewogenes Geschlechterverhältnis angestrebt (drei Studenten, vier Studentinnen). Dies spiegelt bewusst nicht das Geschlechterverhältnis im Studium der Sozialen Arbeit wider, sondern 
ermöglicht einen vergleichsweisen genaueren Blick auf die ,erwartungswidrigen' Wege der männlichen Studenten. Zum anderen wurde eine größtmögliche Vielfalt anvisiert, was die Bildungshintergründe der Befragten und deren mögliche Berufserfahrung vor dem Studium sowie die Bildungshintergründe der Eltern betrifft: Es wurden Studierende mit unterschiedlichen Hochschulzugangsberechtigungen (allgemeine Hochschulreife, Fachhochschulreife) eingeladen, die diese über unterschiedliche Wege (Gymnasium, Gesamtschule, Fachoberschule, Berufsschule) erlangt haben. Zudem sollten Studierende einbezogen werden, die direkt nach ihrem Schulabschluss an die Hochschule wechselten, ebenso wie Studierende, die zwischen Schulabschluss und Studienbeginn unterschiedliche Stationen durchliefen (Freiwilliges Soziales Jahr, Auslandsaufenthalt, Ausbildung, Berufserfahrung).

Was die Bildungshintergründe der Eltern betrifft, sollte ebenfalls eine gewisse Varianz abgebildet werden. Es wurden daher Studierende mit unterschiedlichen elterlichen Hintergründen zum Interview eingeladen (von promovierten Elternteilen über universitäre und fachhochschulische Abschlüsse, allgemeine Hochschulreife, Fachhochschulreife, mittlere Reife, Volksschulabschluss bis hin zu keinem Schulabschluss). Aus bildungs- wie geschlechtertheoretischer Sicht ist es dabei wichtig, den Bildungshintergrund der Eltern differenziert zu betrachten und diesen nicht über den höchsten Bildungsabschluss des Vaters zu erheben. Stattdessen wurden die jeweiligen Bildungsabschlüsse (und Berufe) beider Elternteile ${ }^{4}$ erhoben.

\subsection{Sample und Auswahl der Vergleichsfälle}

Im Zeitraum von November 2015 bis Dezember 2016 wurden dreizehn Interviews mit acht Studierenden der Sozialen Arbeit (fünf davon haben sich als

\footnotetext{
${ }^{4}$ Die Angaben zu den Eltern wurden im Sozialdatenbogen abgefragt. Hierbei konnten die Interviewpartner*innen je Elternteil ankreuzen, ob es sich um „Mutter“, ,Vater“ oder eine andere erziehungsberechtigte Person handelt. Dadurch wurde auch die schriftlichen Datenerhebung hin zu Familienformen jenseits der traditional-binären Form geöffnet. Alle Interviewpartner*innen haben hier „Mutter“ und „Vater“ angegeben, homosexuelle Eltern waren im Sample nicht vertreten. In Fällen von Alleinerziehung wurden zum zweiten (biologischen) Elternteil die Angaben gemacht, die den Befragten bekannt waren. Es sei daher betont, dass diese Angaben keine Aussagen über die tatsächliche soziale Familienkonstellation machen. Erst durch die Erzählungen der Befragten ist ein Einblick darin möglich, welche Rolle Mutter/Vater gespielt haben, ob etwa Alleinerziehung eine Rolle in der Kindheit und Jugend gespielt hat oder ob die Befragten in einer ,Patchworkfamilie“ aufgewachsen sind.
} 
,weiblich` eingeordnet) und fünf Studierenden des Bauingenieurwesens geführt (drei davon haben sich als, weiblich" eingeordnet). Von diesen Interviews wurden elf auf Grundlage von Tonbandaufnahmen vollständig transkribiert ${ }^{5}$. Dabei wurden auch Besonderheiten des Sprechens wie Betonungen, Auslassungen oder Stocken berücksichtigt, um eine habitushermeneutische Analyse zu ermöglichen (vgl. Tabelle 5.1).

Tabelle 5.1 Transkriptionsregeln der Erhebung

\begin{tabular}{l|l}
\hline Transkriptionszeichen & Bedeutung \\
\hline I: & Äußerung der Interviewerin \\
\hline.. & kurze Pause (2-4 Sekunden) \\
\hline$\ldots$ & $\begin{array}{l}\text { längere Pause (ab 5 Sekunden mit } \\
\text { Sekundenangabe) }\end{array}$ \\
\hline unterstrichenes Wort & Betonung \\
\hline GROSSGESCHRIEBENES WORT & laute Aussprache \\
\hline Des-, weil- & Abbruch von Satz oder Wort \\
\hline$(($ schmunzelt)) & $\begin{array}{l}\text { Begleiterscheinung beim Sprechen, bezogen auf } \\
\text { nachfolgende Äußerung }\end{array}$ \\
\hline (...) & Auslassung \\
\hline [Inhalt eckiger Klammer] & $\begin{array}{l}\text { grammatikalische Anpassung oder } \\
\text { Anonymisierung von Orts- oder Personennamen }\end{array}$ \\
\hline
\end{tabular}

Die Auswahl der Vergleichsfälle zur tiefergehenden Analyse erfolge anhand von Kontrastierungen und Vergleichen: Von den dreizehn Fällen wurden nach den ersten Schritten der Auswertung acht Fälle (s. Tabelle 5.2, Fälle mit fett gedruckten Namen) ausführlich habitushermeneutisch interpretiert. Einige dieser Fälle ließen starke Kontraste erahnen, was die habitusspezifischen Muster der Studienfachwahlen angeht, während andere auf feine Nuancen - etwa innerhalb eines ähnlichen Milieus, aber vor dem Hintergrund verschiedener Prozesse der Vergeschlechtlichung - verwiesen. Diese Fälle werden in Kapitel 6 ausführlich dargestellt. Die Reihenfolge dieser Darstellung orientiert sich an einem Wechsel von Kontrasten und ähnlich gelagerten Fällen: So können die Fälle „Sonja“

\footnotetext{
${ }^{5}$ Auf die vollständige Transkription des Falls „Viktor“ wurde verzichtet, da dieser Fall aus der weiteren Analyse ausgeklammert wurde (s. nächste Fußnote). Ein weiteres Interview war als Pre-Test angelegt, um den Leitfaden $\mathrm{zu}$ erproben; dieses wurde weder aufgezeichnet noch transkribiert und wird deshalb in der Falltabelle nicht aufgeführt.
} 
und „Tabea“ als Kontrastfälle gesehen werden, ebenso wie „Achim“ und „Michael“, während der dritte ,männliche“ Fall „Dominic“ zwischen diesen beiden zu verorten ist. „Rebecca“ wiederum zeigt einige Parallelen zu „Dominic“, aber auch bedeutsame Unterschiede. ,Jennifer“ und „Hanna“ dagegen weisen relativ ähnliche Habitusmuster auf, die jedoch zu unterschiedlichen Fachwahlen führen.

Die vier Fälle, die keiner ausführlichen Analyse unterzogen wurden, lagen entweder anderen Fällen sehr nah und versprachen somit wenig kontrastive Erkenntnisse (Lilli und Ben) oder das Interviewmaterial zentrierte sich um eine weitere Dimension jenseits von Klasse und Geschlecht („Bahar“: Religion, „Viktor“: Migrationshintergrund), weshalb sie aus Gründen der besseren Vergleichbarkeit nicht näher einbezogen wurden. Im Rahmen einer intersektionalen Analyse, die sich nicht - wie hier - auf die Dimensionen von Klasse und Geschlecht fokussiert, wäre eine Analyse dieser beiden Fälle sehr vielversprechend.

Ganz allgemein ist festzustellen, dass im Sample zwar eine gewisse Varianz hinsichtlich der Bildungshintergründe der Befragten abgebildet ist, diese aber durchaus beschränkt ist: Das Sample lässt sich grundsätzlich als ,bildungsnah' beschreiben, Elternteile mit Volksschulabschluss oder ohne Schulabschluss sind die Ausnahme. Auch die Befragten selbst haben bereits einen (schulischen) Selektionsprozess durchlaufen und die Hochschulreife absolviert.

\section{$5.4 \quad$ Interviewführung}

\subsubsection{Konzeption der Interviewführung}

Die Konzeptionierung der Interviewführung lehnt sich an die Perspektive Bourdieus (insb. Bourdieu 1997c) und Jean-Claude Kaufmanns, insbesondere seine verstehende Interviewführung, an (Kaufmann 1999). Bourdieu versteht jede Befragungssituation als soziale Beziehung, die von entsprechenden Hierarchieverhältnissen und Verzerrungen geprägt ist. Diese verzerrenden Effekte müssen nach Bourdieu erkannt und weitestgehend kontrolliert werden, vor allem durch Reflexivität. Reflektiert werden muss insbesondere die bestehende Hierarchie zwischen Interviewer*in und Befragten: Zum einen gibt die interviewende Person Gegenstand und Rahmenbedingungen der Befragung vor und bestimmt damit die Grundlagen der Interaktion. Zum anderen besteht üblicherweise ein Gefälle zwischen Interviewer*in und Befragten, was den Umfang von kulturellem Kapital angeht. Diese Asymmetrie muss nach Bourdieu anerkannt werden, um die symbolische Gewalt, die durch die interviewende Person ausgeübt wird, möglichst $\mathrm{zu}$ reduzieren. Ein Ausschalten der verzerrenden Effekte hingegen ist 


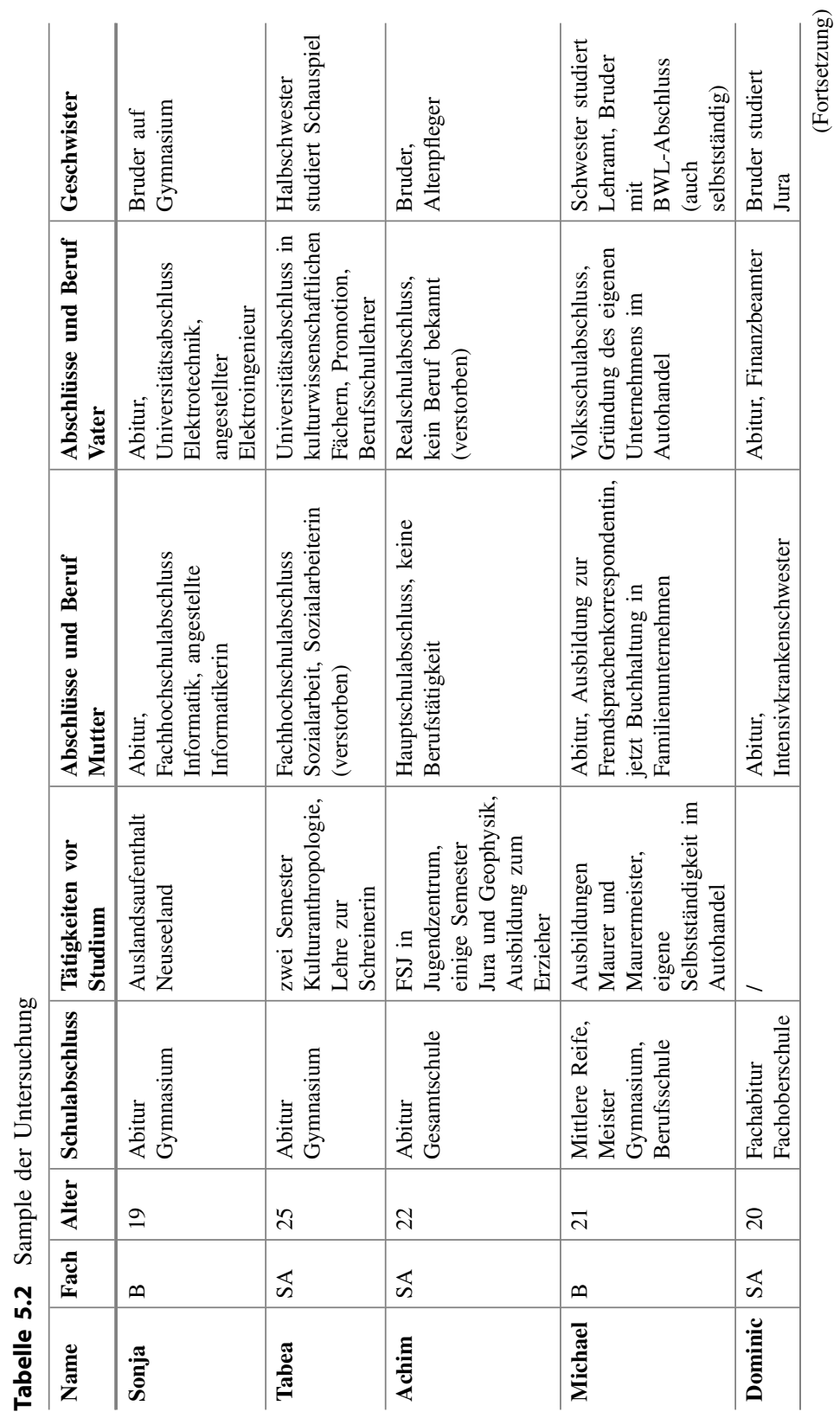




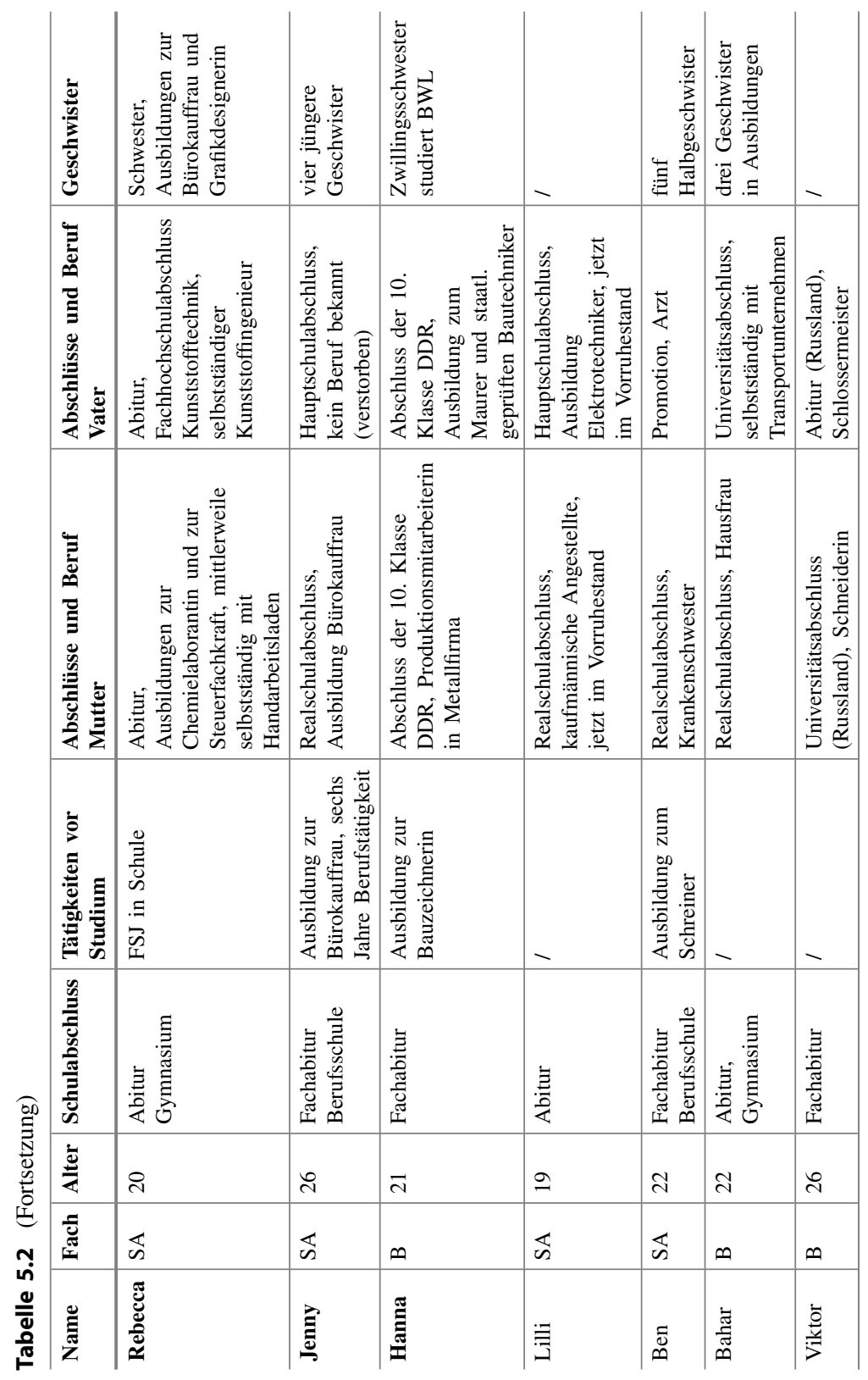


nicht möglich - eine Befragungssituation ist also nie ,neutral' (Bourdieu 1997c: 780 ff.).

Diese Asymmetrie war in der vorliegenden Befragung von Studierenden des ersten Semesters zwar vorhanden, kann aber einerseits als moderat eingeschätzt werden: So war die Interviewerin zum einen nur wenige Jahre älter als die Befragten und zum anderen befanden sich die Befragten in einer akademischen Ausbildung, während die Interviewerin die gleiche bzw. eine vergleichbare Ausbildung abgeschlossen hatte. Andererseits muss davon ausgegangen werden, dass eine gewisse Hierarchie die Interviewsituation geprägt hat - schließlich wurden Studierende durch eine Doktorandin interviewt und es mögen sich zudem Unterschiede im Auftreten niedergeschlagen haben. Diese Asymmetrie muss in der Auswertung des Materials berücksichtigt werden.

Die tendenzielle Nähe von Interviewerin und Befragten hat sich als gute Grundlage erwiesen, um eine möglichst lockere und aus Perspektive der Befragten entspannte Situation zu schaffen, in der sich die Befragten treiben lassen konnten (Kaufmann 1999: 91). So sollte das Interview zu einem möglichst intensiven Austausch werden, um an die wesentlichen Aussagen heranzukommen, ohne dabei in ein Gespräch abzudriften (ebd.: 70). Dazu hörte die Interviewerin aktiv und methodisch zu und passte sich dabei auch der befragten Person an, indem sie ihre Sprache übernahm sich ihre Sichtweisen zu eigen machte (Bourdieu 1997c: 784). Um die Interviewsituation vor diesem Hintergrund möglichst gesprächsnah zu gestalten, wurden Kekse bereitgelegt und Kaffee angeboten. Außerdem duzten sich beide Beteiligten. Die Gespräche fanden in einem Büroraum auf dem Campus der Hochschule statt, an der die Befragten studierten. Das mag durchaus einen Einfluss auf die Gesprächsatmosphäre gehabt haben, schließlich nahmen die Befragten die Interviewerin so als Mitarbeiterin der Hochschule wahr, was etwa ein teilweise formelleres Antwortverhalten bedingen mochte. Telefonische Unterbrechungen wurden durch Stummschalten des Apparates und persönliche Unterbrechungen durch einen entsprechenden Hinweis an der geschlossenen Bürotür vermieden. Vor den Interviews wurden die Befragten nochmals über die Anonymisierung des Materials und die folgende Tonbandaufnahme aufgeklärt. Die Interviews dauerten zwischen 45 und 90 Minuten. 


\subsubsection{Leitfadengestützte themenzentrierte Interviews}

Die Befragungen wurden als leitfadengestützte themenzentrierte Interviews ${ }^{6}$ gestaltet. Der Leitfaden (s. Anhang) diente weniger einer starren Vorgabe als viel mehr einer flexiblen Orientierungshilfe mit mehreren Themenblöcken und einigen konkreten, offenen Fragen im Sinne von Erzählaufforderungen (Kaufmann 1999: $65 \mathrm{ff}$.). Im Leitfaden und im Interview wurde die Studien(fach)wahl nicht als isoliertes Thema, sondern eingebettet in die gesamte Lebensführung betrachtet (s. Abschnitt 3.3.2). Um auch die Dimension von Geschlecht analytisch fassen zu können, wurden etwa vergeschlechtlichte Praktiken in der Herkunftsfamilie thematisiert (Vorbilder, Berufe, Erziehungsorganisation) und die Studien(fach)wahl im Kontext der Gesamtheit an vergeschlechtlichten Habituspraktiken betrachtet, indem in den Interviews auch die Antizipation von Familiengründung und -organisation abgefragt wurde.

Als Einstieg wurde in jedem Interview die gleiche Erzählaufforderung gegeben: „Erzähl doch mal ganz ausführlich, wie es dazu gekommen ist, dass du dich für Soziale Arbeit/ Bauingenieurwesen entschieden hast!“. Anschließend folgte ein Themenblock zur „Habitusformierung“ (in Anlehnung an Schneickert 2013: 199), der meist mit der Frage eingeleitet wurde „Wie finden deine Eltern deine Studienfachwahl?“ eingeleitet wurde, um dann auf Kindheit, Schulzeit usw. einzugehen. Danach wurde das aktuelle Studium thematisiert, beginnend mit der Frage: „Wie würdest du dein erstes Semester bisher beschreiben?“. Und schließlich wurde ein Austausch über die (berufliche) Zukunft angestoßen, indem die Befragten erzählen sollten: „Stell dir dein Leben in zehn Jahren vor - wie soll das aussehen?". Als Abschluss wurden in den meisten Interviews folgende zwei Fragen gestellt:

- Eine ganz allgemeine Frage: Worauf kommt es Dir im Leben an?

- Stell Dir vor, Du hättest drei Wünsche frei, was würdest Du Dir wünschen?

Diese zwei „Standardfragen“ haben sich in der Milieuforschung als besonders erkenntnisreich erwiesen (Teiwes-Kügler/Lange-Vester 2018: 129). So bilden sich gerade ,[i]n der Auseinandersetzung z. B. in Fragen, was ,richtig' und was ,falsch" im Leben ist, was erstrebenswert ist und was als unwichtig vernachlässigt werden kann, (...) Positionen und Lebensentwürfe und mit ihnen der

\footnotetext{
${ }^{6}$ Zur Methodologie und Durchführung qualitativer Interviews allgemein siehe weiterführend auch Helfferich (2011) und Misoch (2019).
} 
Habitus heraus“ (Bremer/Teiwes-Kügler 2013a: 205). Erzählungen der Befragten darüber, worauf es ihnen im Leben ankäme und wie sie sich ihre Zukunft vorstellen, sind also besonders hilfreiche Hinweise auf ihren vergeschlechtlichten Milieuhabitus - auch und gerade im Kontext ihrer Studien(fach)wahl.

Bis auf die Eingangssequenz und die Abschlussfragen divergierte die Reihenfolge der Themenblöcke zwischen den Interviews. Die Interviewführung orientierte sich an den Erzählungen und Impulsen der Befragten und knüpfte an bereits Gesagtes an. So ergaben sich unterschiedliche thematische Reihenfolgen und Schwerpunkte. Die thematisierten Bereiche spiegeln sich auch in den Falldarstellungen (s. Kapitel 6) wider: So werden dort alle Fälle anhand der übergeordneten Themenblöcke „Kurzeinführung in den Fall“, „Studien(fach)wahl im Kontext der Herkunftsfamilie“, „Stationen vor dem Studium“ und „Studien(fach)wahl und antizipierte Zukunft" dargestellt. Außerdem wird jeder Fall eingeleitet mit der „Reflexion der Interviewsituation“, um Besonderheiten im Gespräch und atmosphärische Randbedingungen widerzuspiegeln.

Am Ende des Interviews füllten die Befragten einen Sozialdatenbogen aus und konnten detaillierte Angaben machen, in welcher Form das Interviewmaterial (Aufnahme, Transkription, Fallanalysen) verwendet werden darf und anonymisiert werden soll.

\subsection{Interviewauswertung}

\subsubsection{Methode der Habitushermeneutik}

Bourdieu hat zwar - etwa in „Die feinen Unterschiede“ (1982) - eine differenzierte Beschreibung von unterschiedlichen, klassenspezifischen Habitus vorgenommen, allerdings kein fertiges methodisches Instrument hinterlassen, um den Habitus zu entschlüsseln. Diese Lücke wird u. a. von der seit den 1990er-Jahren entwickelten „Habitushermeneutik“ (etwa Bremer/TeiwesKügler 2013b; Bremer/Teiwes-Kügler 2013a; Lange-Vester/Teiwes-Kügler 2013a; Teiwes-Kügler/Lange-Vester 2018) geschlossen, die auf der Milieuforschung nach Vester basiert und damit ebenso an Bourdieu anschließt ${ }^{7}$.

\footnotetext{
${ }^{7}$ Ein anderes Verfahren zur Habitusanalyse ist etwa jenes nach der Dokumentarischen Methode (vgl. etwa Bohnsack 2013; empirisch angewendet etwa von Kramer 2014). Der besondere Erkenntnisgewinn der Habitushermeneutik liegt jedoch u. a. in ihrem Bezug zur Typologie sozialer Milieus (nach Vester 2001), weshalb sie sich für die vorliegende Arbeit als besonders anschlussfähig erweist.
} 
Der Begriff der Habitushermeneutik entstand aus dem Forschungsprozess heraus und drückt aus, dass mit dem ,Lesen' und, Verstehen' des Habitus eine spezifische Deutung verknüpft ist. So wie sich klassenspezifische Praktiken nach Bourdieu nicht aus Statistiken schließen lassen, lässt sich dementsprechend auch der Habitus nicht aus sozialen Positionen oder Kapitalien ableiten - sondern:

„Er muss aus den Mustern der sozialen Praxis erschlossen werden. Als handlungsorganisierendes Prinzip ('modus operandi') hinterlässt er in den Praktiken eines Akteurs eine bestimmte Handschrift, die jedoch in den Praxisformen codiert enthalten ist und durch Interpretationsarbeit entschlüsselt werden muss" (Bremer/Teiwes-Kügler 2013b: 93).

Die Habituszüge der Akteur*innen sind dabei eng verbunden mit ihrem sozialen Milieu:

„Praxis und Haltungen der Milieuangehörigen werden nicht einfach als ein beliebiger Ausdruck persönlicher Willenskraft aufgefasst. Vielmehr folgt die Milieuanalyse dem Verständnis, dass Neigungen und Auffassungen, die Menschen haben, in aktiver Auseinandersetzung mit sozialen Positionierungen, Ressourcen und damit verbundenen Möglichkeiten, bestimmte Ziele anzustreben und zu erreichen, entwickelt und eingeprägt werden“" (Teiwes-Kügler/Lange-Vester 2018: 113).

So kann mit der Habitushermeneutik auch eine typologische Differenzierung vorgenommen werden entlang der Wahrnehmungs-, Denk- und Handlungsmustern sozialer Milieus (Lange-Vester/Teiwes-Kügler 2013a: 156), wie es bereits in mehreren Forschungsprojekten - etwa zum Feld von Hochschule und Studium (s. Abschnitt 3.3.2.3) - vorgenommen wurde. Es eignen sich insbesondere lebensgeschichtliche und themenzentrierte Interviews sowie Gruppenverfahren wie mehrstufige Gruppenwerkstätten zur Entwicklung von Material, aus dem der Habitus hermeneutisch entschlüsselt werden kann. Das interpretative Vorgehen gleicht einem ,kriminalistischen Sammeln von Indizien, die am Ende der Auswertung zu einem plausiblen, aber nicht unbedingt widerspruchsfreien Gesamtbild - dem Habitussyndrom - der befragten Person zusammengefügt werden müssen“ (Bremer/Teiwes-Kügler 2013a: 210). Wie es das Konzept des Habitus nahelegt, werden beim habitushermeneutischen Vorgehen zwei Sinnschichten sozialer Praxis unterschieden: eine primäre Sinnschicht (offenkundiger Sinn) und eine sekundäre Sinnschicht (latenter Sinn). Die Analyse von z. B. Interviewmaterial beruht auf der Annahme, dass sich Akteur*innen ,im Alltag durch Bewertungen ständig implizit zuordnen und abgrenzen, und dass diese Prozesse mit den gesellschaftlichen Teilungen zusammenhängen“ (Bremer/Teiwes-Kügler 
2013b: 113). Diese alltägliche Strukturierungsarbeit beruht auf klassifizierenden Gegensatzpaaren, auf die bereits Bourdieu hinweist: hoch - niedrig, leicht schwer, spirituell - materiell usw. (Bourdieu 1982: 730). Im Rahmen der Milieuforschung um Vester und anderen wurde daraus empirisch ein Kategoriensystem entwickelt, um Praktiken zu begreifen und für die Milieuanalyse nutzbar zu machen. Ergebnis zahlreicher Untersuchungen ist eine heuristische Synopse an Elementarkategorien (s. Tabelle 5.3). Diese Kategorien können als ,elementare Dimensionen des Habitus“ (Bremer/Teiwes-Kügler 2013b: 113) verstanden werden im Sinne von gegensätzlichen (und idealtypischen) Polen. In der Realität setzt sich der Habitus zusammen aus mehreren Dimensionen bzw. Zügen, die jeweils in die eine oder andere Richtung des polaren Gegensatzes tendieren. Jede Elementarkategorie wird durch einen ,Hauptbegriff“ betitelt (bspw. ,asketisch“) und durch weitere ,Unterbegriffe" näher umschrieben (,methodisch“; „planend“; „Pflicht“...) (ebd.: 114). Diese Unterbegriffe konkretisieren mögliche Ausdrucksformen dieses Habituszugs (ebd.).

Das besondere Erkenntnispotential der heuristischen Elementarkategorien liegt also darin, dass die Bourdieusche Annahme von der Inkorporation sozialer Strukturen als kognitive Strukturen (Bourdieu 1982: 730) empirisch fundiert und daraus ein analytisches Raster entwickelt wird. Zugleich sind die Elementarkategorien nicht universell oder erschöpfend. Was den Einbezug dieser Elementarkategorien in den Interpretationsvorgang angeht, soll betont werden, dass „, [d]ie Arbeit mit theoretischen Kategorien (...) nicht davon entlastet, die Logik jeweils aus dem Fall heraus zu rekonstruieren; sie hilft aber, sich dem sozialen Ort des jeweiligen Falls anzunähern und die Bedingungen und Möglichkeiten auszuloten, die für diesen bestehen“ (Teiwes-Kügler/Lange-Vester 2018: 133). Deshalb werden die Elementarkategorien erst relativ spät im Interpretationsprozess hinzugezogen, nämlich dann, wenn dieser bereits anhand mehrerer Passagen sequenzanalytisch fortgeschritten ist und den Spuren des Habitus so bereits induktiv nachgegangen wurde. Die heuristischen Kategorien dienen folgendermaßen dazu, die gefundenen Habituszüge begrifflich zu konturieren und werden nicht als deduktives Raster an das Material angelegt ${ }^{8}$. Zudem gilt es, die relevanten Habitusdimensionen für jedes Feld neu herauszuarbeiten bzw. zu erweitern (Bremer/Teiwes-Kügler 2013b: 114).

\footnotetext{
${ }^{8}$ Bisweilen wird der Habitushermeneutik durch ihren Einbezug von Elementarkategorien ein ,subsumptionslogisches Vorgehen“ (Kramer 2018: 246) vorgeworfen, was jedoch durch die späte und konturierende statt definierende Bezugnahme entkräftet werden kann. Gleichzeitig ist ebenjener Einbezug von theoretischen Kategorien eine besondere Stärke der Methode (vgl. ebenso Kramer 2018), die so der Strukturiertheit des Habitus Rechnung trägt.
} 
Tabelle 5.3 heuristische Synopse aus den Projekten „Soziale Milieus im gesellschaftlichen Strukturwandel“, „Kirche und Milieu“, „Studierendenmilieus in den Sozialwissenschaften"

\begin{tabular}{|c|c|}
\hline $\begin{array}{l}\text { asketisch } \\
\text { methodisch; planend; Pflicht; (Trieb-) } \\
\text { Verzicht steht vor Lust und Genuss; } \\
\text { diszipliniert; Selbstbeherrschung }\end{array}$ & $\begin{array}{l}\text { hedonistisch } \\
\text { spontan; ungeplant; ungeregelt; lustbetont; } \\
\text { Spaß; Lust und Genuss statt Pflicht und } \\
\text { Verzicht; Erlebnisorientierung }\end{array}$ \\
\hline $\begin{array}{l}\text { ideell } \\
\text { spirituell; metaphysisch; Neigung zur } \\
\text { Abstrahierung von der dinglichen } \\
\text { Realität; vergeistigt; intellektuell; } \\
\text { idealistisch; ,Betonen des Anspruchs } \\
\text { auf Authentizität‘ }\end{array}$ & $\begin{array}{l}\text { materiell } \\
\text { körperbetont; ,weltlich“; praktisch; } \\
\text { Orientierung am konkret Fassbaren; } \\
\text { verdinglicht; realistisch; Pragmatismus: } \\
\text { Orientierung an Machbarkeit und } \\
\text { Notwendigkeit }\end{array}$ \\
\hline $\begin{array}{l}\text { hisch } \\
\text { tsorientiert bis autoritär; } \\
\text { enken; positive Bewertung von } \\
\text { g und Unterordnung; häufig: } \\
\text { iments }\end{array}$ & $\begin{array}{l}\text { egalitär } \\
\text { partnerschaftlich; demokratisch; Anspruch } \\
\text { auf Gleichberechtigung, Partizipation und } \\
\text { Mitgestaltung; integrativ; ,leben und } \\
\text { leben lassen“ }\end{array}$ \\
\hline $\begin{array}{l}\text { individuell } \\
\text { Vorrang des Selbst vor der } \\
\text { Gemeinschaft; Autonomie: Anspruch } \\
\text { auf Unabhängigkeit und } \\
\text { Selbstbestimmung (,jeder ist für sich } \\
\text { selbst verantwortlich`); häufig Streben } \\
\text { nach Selbstverwirklichung und } \\
\text { Persönlichkeitsentfaltung; Neigung zu } \\
\text { Egozentrik; abgrenzen von der } \\
\text {,Masse‘, Betonung von } \\
\text {,Einzigartigkeit‘ und } \\
\text { Unkonventionalität }\end{array}$ & $\begin{array}{l}\text { gemeinschaftlich } \\
\text { Gemeinschaft steht vor individuellen } \\
\text { Ansprüchen; Rücksichtnahme auf } \\
\text { Konventionen; Bereitschaft zu } \\
\text { Kompromissen; teilweise Anpassung und } \\
\text { Konformismus; Geselligkeit, Sicherheit, } \\
\text { und Geborgenheit; bisweilen Anlehnung } \\
\text { an bzw. Entlastung durch die } \\
\text { Gemeinschaft }\end{array}$ \\
\hline $\begin{array}{l}\text { ästhetisch } \\
\text { Form steht vor Inhalt; Vorrang der } \\
\text { Ästhetik vor Funktionalität; } \\
\text { Distanzierung von unmittelbaren und } \\
\text { direkten Ausdrucksformen; Stilisierung } \\
\text { von Praktiken; Betonung des ,Schönen“ } \\
\text { und Stilvollen gegenüber Nützlichkeit } \\
\text { und Zweckmäßigkeit; Feingeschmack }\end{array}$ & $\begin{array}{l}\text { funktional } \\
\text { Inhalt ist wichtiger als Form; Orientierung } \\
\text { an Funktionalität; Zweckmäßigkeit und } \\
\text { Nützlichkeit stehen im Vordergrund; } \\
\text { unmittelbare und direkte } \\
\text { Ausdrucksformen herrschen vor; } \\
\text { Notwendigkeits- oder Grobgeschmack }\end{array}$ \\
\hline
\end{tabular}

(Fortsetzung) 
Tabelle 5.3 (Fortsetzung)

\begin{tabular}{|c|c|}
\hline $\begin{array}{l}\text { aufstiegsorientiert } \\
\text { Streben nach ,Höherem“; Karriere- und } \\
\text { Statusorientierung; } \\
\text { konkurrenzorientiert, z. T. } \\
\text { kalkülbetontes Verhalten und } \\
\text { Ellenbogenmentalität; z. T. Auf- bzw. } \\
\text { Abstiegsängste }\end{array}$ & $\begin{array}{l}\text { sicherheitsorientiert } \\
\text { „Lieber den Spatz in der Hand als die } \\
\text { Taube auf dem Dach“; realistischer Sinn } \\
\text { für die eigenen Grenzen; geringe } \\
\text { Risikobereitschaft; Festhalten an } \\
\text { Vertrautem und Gewohntem; „Jeder sollte } \\
\text { an seinem Platz bleiben und das Beste } \\
\text { daraus machen“ }\end{array}$ \\
\hline $\begin{array}{l}\text { herrschend } \\
\text { Machtansprüche; Dominanz; sozialer } \\
\text { Blick von oben nach unten; z. T. } \\
\text { karitativ; z. T. offen ausgrenzend und } \\
\text { elitär; symbolische Formen der } \\
\text { Herrschaft über hochkulturelle Muster }\end{array}$ & $\begin{array}{l}\text { ohnmächtig } \\
\text { Fatalismus; sich dem Schicksal } \\
\text { ausgeliefert fühlen; dichotomes Weltbild; } \\
\text { sozialer Blick von unten nach oben }\end{array}$ \\
\hline $\begin{array}{l}\text { selbstsicher } \\
\text { selbstbewusst; Selbstgewissheit im } \\
\text { Umgang mit Anforderungen; } \\
\text { Anspruchshaltung; meist zielsicher; } \\
\text { Zukunftsoptimismus }\end{array}$ & $\begin{array}{l}\text { unsicher } \\
\text { Selbstzweifel u. wenig Selbstvertrauen in } \\
\text { nicht vertrauten Feldern; soziale Distanz } \\
\text { zu Autoritäten; wenig Zuversicht, neue } \\
\text { Anforderungen bewältigen zu können; } \\
\text { häufig Skepsis bis Pessimismus } \\
\text { hinsichtlich der eigenen Zukunft }\end{array}$ \\
\hline
\end{tabular}

Quelle: Teiwes-Kügler/Lange-Vester 2018: 133 f.

Das habitushermeneutische Vorgehen sieht konkret vier methodische Auswertungsschritte vor (Bremer/Teiwes-Kügler 2013a: $207 \mathrm{ff}$.):

1. Es wird ein analytisches Verlaufsprotokoll verfasst. Welche Themen und Episoden in welchem Umfang als erzählenswert betrachtet werden, unterscheidet sich je nach Milieu. Deshalb gilt es, den thematischen Verlauf des Interviews komprimiert herauszuarbeiten und einen Überblick über die inhaltlich-thematischen Schwerpunkte zu geben.

2. Auf Grundlage von Sequenzanalysen wird die hermeneutische Interpretation des Materials vorgenommen, um die latenten Sinn- und Bedeutungszusammenhänge im Material offenzulegen. Dazu wird üblicherweise die Einstiegssequenz als Erstes betrachtet. Die Interpretationsarbeit erfolgt in möglichst vielfältig besetzten Gruppen, um unterschiedliche Lesarten zu entwickeln. Dieses sequenzanalytische Vorgehen wird an ausgewählten, an der Forschungsfrage 
orientierten Passagen durchgeführt. Im weiteren Verlauf der Interpretation werden so anhand des konkreten Materials abstrahierende Deutungen vorgenommen und dabei in heuristischer Weise auch auf das Kategoriensystem zurückgegriffen - dabei werden einige Lesarten verworfen, ergänzt oder sie erhärten und bestätigen sich. Diese Lesarten können als Spuren des Habitus gesehen werden.

3. Im dritten Schritt wird das Habitus-Syndrom gebildet. Hier geht es nicht darum, die einzelnen herausgearbeiteten Habituszüge zu addieren oder einen einzelnen Zug als ,typisch ‘ für ein Milieu zu interpretieren, sondern es lässt sich aus spezifischen Verbindungen von Habituszügen auf ein mögliches Milieu schließen.

4. Im letzten Schritt werden schließlich für das untersuchte gesellschaftliche Feld die entsprechenden ,Habitustypen' gebildet. Dies erfolgt durch eine komparative Analyse und die Gruppierung ähnlicher Fälle sowie eine vergleichende Einordnung der Typen in den Raum der sozialen Milieus.

Was die vorliegende Untersuchung zu Studien(fach)wahlen angeht, wurde das beschriebene habitushermeneutische Vorgehen bei acht der dreizehn geführten Interviews im Zeitraum von Februar 2016 bis Mai 2018 durchgeführt. Dazu wurden die Transkripte in mehreren unterschiedlichen Gruppen gemeinsam interpretiert, mögliche Lesarten gebildet, geprüft und teilweise verworfen, bis sich für jeden Fall erste Spuren des Habitus eröffneten, die in Einzelarbeit weiterbearbeitet und dann wieder in Gruppenkontexte zur gemeinsamen Interpretation und Reflexion zurückgespielt wurden. Die Auswahl der interpretierten Sequenzen orientierte sich an der Forschungsfrage, wie die Studien(fach)wahl im Kontext von Geschlecht und sozialem Milieu nachvollzogen werden kann und umschließt daher mehrere relevante Passagen, welche im Folgenden erläutert werden.

Die Einstiegssequenz wurde in allen Interviews ausführlich interpretiert, da sie bereits entscheidende Hinweise auf die habitusspezifischen Muster der Studien(fach)wahl gibt. Zusätzlich wurden einzelne Passagen zur Kindheit und Schulzeit und im Regelfall auch die Darstellung der Sichtweise der Eltern auf die Studien(fach)wahl analysiert. Auch verschiedene Passagen zu Zukunftsvorstellungen inklusive den Berufsvorstellungen, Überlegungen zur Partnerschaft, Familienplanung und der Organisation von Arbeit und Familie wurden ausgewertet. Häufig wurden diese Passagen abgeglichen mit den Erfahrungen der Befragten in ihrer jeweiligen Herkunftsfamilie - mit den Entstehungsbedingungen des vergeschlechtlichen Habitus also. So ergibt sich ein umfangreiches Bild darüber, welche Pläne, Motive und Ziele die Befragten mit ihrem Studium verbinden, welcher Weg dem vorausgegangen ist, welche bildungsspezifischen und vergeschlechtlichten 
Praktiken sich in der Herkunftsfamilie andeuten und welche Zukunft die Person als Mann bzw. Frau eines spezifischen sozialen Milieus antizipiert. Da viele dieser habitusspezifischen Muster der Studien(fach)wahl auf einer eher unbewussten, vorreflexiven Ebene verankert sind, bietet sich das genannte Verfahren der Habitushermeneutik an, um auch diese latente Ebene zu entschlüsseln.

Die ,Verortung' des jeweiligen Falls im Raum der sozialen Milieus geschieht in der folgenden Analyse zum einen vor der allgemeinen Hintergrundfolie der repräsentativen Makromilieus (Vester 2001; Vester 2015) und zum anderen mit Rückgriff auf die Milieuanalysen zu Studierenden (insbesondere LangeVester/Teiwes-Kügler 2004; Hild 2019; Grunau 2017).

\subsubsection{Zum methodischen Umgang mit Geschlecht}

\subsubsection{Geschlecht als empirische Kategorie}

In der habitushermeneutischen Interpretation des Interviewmaterials wurden sowohl die Dimension von Klasse wie auch Geschlecht berücksichtigt, um klassenspezifische Prozesse der Vergeschlechtlichung im Kontext der Studien- und Berufswahl zu entschlüsseln. Dieses - im Rahmen der Habitushermeneutik neuartige - Vorgehen verweist auf ein bekanntes Problem der Geschlechterforschung: dem empirischen Umgang mit Geschlecht ${ }^{9}$. Dieses Problem umfasst im Kontext des vorliegenden Forschungsprojekts insbesondere drei Aspekte: Die Reifizierung von Geschlecht, die Setzung von Geschlecht als Leitdifferenz und die Erfassung von Geschlecht als etwas ,fraglos Gegebenes'. Diese drei Aspekte werden im Folgenden detailliert erläutert.

\section{Die Reifizierung von Geschlecht}

Seit der sozialkonstruktivistischen Wende der Geschlechterforschung und der damit verbundenen Setzung von Geschlecht als Ergebnis sozialer Konstruktionsprozesse wird regelmäßig die Frage gestellt, wie Geschlecht empirisch betrachtet werden kann, ohne sich zugleich an seiner Konstruktion zu beteiligen - wie also die Reifizierung von Geschlecht dabei vermieden werden kann:

„Einer der Tücken dieses zweigeschlechtlichen Klassifikationsverfahrens besteht darin, daß man es kaum beschreiben, kaum rekonstruieren kann, ohne es - zumindest hinterrücks und indirekt - selbst nachzuvollziehen und damit erneut zu bestätigen und zu reifizieren“ (Wetterer 1995: 22 f.).

\footnotetext{
${ }^{9}$ Siehe weiterführend zum methodologischen „Nachdenken über die Kategorie Geschlecht“ etwa Althoff et al. (2017: 417 ff.).
} 
Um sich einer Rekonstruktion ohne Reifizierung anzunähern, muss daher auf die Prozesse der Vergeschlechtlichung und damit den Prozesscharakter von Geschlecht im Vollzug sozialen Handelns geblickt werden (Wetterer 1995: 22 f.). Im Rahmen dieses Forschungsprojekts wird die Annäherung dieses Ziels, nämlich Geschlecht nicht unreflektiert zu reifizieren, durch folgende drei Aspekte im empirischen Vorgehen sicher gestellt:

Erstens wurde das Geschlecht der Befragten durch eine Selbstzuordnung erhoben, in der Regel in Form von einer Fragebogenabfrage, die mehr Angaben zuließ als die binäre Aufteilung in ,weiblich“ und ,männlich`. Wenn in der Ergebnisdarstellung also von Fällen mit kulturell eindeutig weiblichen oder männlichen Namen die Rede ist, beruht das auf den Angaben der Befragten.

Zweitens wurde das Material zur Interpretation in Gruppen so anonymisiert, dass das soziale Geschlecht der Befragten (im Sinne ihrer Selbstzuordnung) nicht direkt ersichtlich war - etwa durch ein vergeschlechtlichtes Alias oder eindeutige Aussagen der Befragten. Die Interpretation des Interviews wurde so weniger gelenkt und konnte unvoreingenommener durchgeführt werden. ${ }^{10}$ Dabei zeigte sich, dass in den Interpretationsgruppen häufig eine Dynamik entstand, das Geschlecht der Befragten ,entlarven`zu wollen - dieser Wunsch nach der eindeutigen Zuordnung der Geschlechtszugehörigkeit lässt sich als immanenter Bestandteil des gesellschaftlichen Systems der Zweigeschlechtlichkeit fassen (vgl. etwa West/Zimmerman 1987). Hier wurde also durchaus eine bewusste Reifizierung von Geschlecht vorgenommen, allerdings unter ständiger (Selbst-)Reflexion und Irritation. Damit verbunden war die Erkenntnis, dass diese Versuche der sozialen ,Zuordnung' auf der Grundlage der Erzählungen der Befragten alles andere als widerspruchsfrei verliefen - der überwiegende Teil der Interviews war von Uneindeutigkeiten geprägt, was potenzielle Zuschreibungsprozesse angeht. Häufig wurden erst bei der Thematik von Familienplanung konkrete Vermutungen über die soziale Geschlechtszugehörigkeit der Befragten geäußert. Das lässt darauf schließen, dass sich das kulturelle Wissen über die Binarität von Geschlecht durch entsprechendes methodisches Vorgehen nachhaltig irritieren lässt und Zuordnungen damit nicht mehr so einfach sind, wie sie es im Alltagswissen sein mögen - was dort als, weiblich 'oder , männlich' gilt, verliert bei näherer Betrachtung an Eindeutigkeit. Durch diese offene und ungerahmte Interpretation des Interviewmaterials eröffnet sich also der Blick hinter die geschlechtliche Binarität und es rücken, je nach Passage und damit je nach Kontext, auch andere

\footnotetext{
${ }^{10}$ Die Forscherin, die das Geschlecht der Befragten natürlich selbst kannte, hat sich bei diesen Interpretationen vorerst zurückgehalten, um ebenfalls nicht lenkend einzugreifen.
} 
Dimensionen in den Vordergrund, wie durch die Analysen noch deutlicher werden wird.

Schließlich wird drittens in der Analyse selbst nicht der Frage nachgegangen, inwiefern sich im Material Hinweise auf einen vermeintlichen, weiblichen' oder ,männlichen' Habitus zeigen, etwa indem bestimmte Habituszüge binär codiert werden (bspw. in der der Konnotation eines fürsorglichen, gemeinschaftlichen Habituszugs als, weiblich'). Stattdessen wird in der Analyse die Prozesshaftigkeit der Vergeschlechtlichung von Studien(fach)wahlen betrachtet. Indem nicht die Frage nach ,weiblichem' oder ,männlichem' habitusspezifischen Handeln gestellt wird, sondern nach der Vergeschlechtlichung des Handelns, wird der Konstruiertheit von Geschlecht Rechnung getragen und das Risiko der Reifizierung zumindest erheblich reduziert.

\section{Die Setzung von Geschlecht als Leitdifferenz}

Das Paradigma der Intersektionalität, dessen Ursprung in der Schwarzen Frauenrechtsbewegung der USA verortet werden kann (Lutz/Vivar/Supik 2013a: 10), hat mittlerweile breiten Einzug in die empirische Geschlechterforschung gefunden. Jedoch hat der Forschungsstand zu Fragen nach der Vergeschlechtlichung von Studien(fach)- und Berufswahlen gezeigt, dass hier in einigen Ansätzen ausschließlich oder schwerpunktmäßig nach der Bedeutung von Geschlecht gefragt wird und insbesondere die Bedeutung der sozialen Herkunft häufig vernachlässigt wird. Indem ausschließlich erforscht wird, wie ,Frauen' ihre Wege in die Ingenieurwissenschaften finden oder ,Männer' in die Soziale Arbeit, wird Geschlecht zur Leitdifferenz erhoben. In der vorliegenden Untersuchung soll dies durch zwei Strategien umgangen werden: Zum einen wurde im Gespräch mit den Befragten das Geschlecht nicht als relevant gesetzt ${ }^{11}$. Nur wenn die Befragten selbst aktiv auf die Dimension von Geschlecht zu sprechen kamen, wurde daran angesetzt und nachgefragt. So sollte vermieden werden, den Befragten die Relevanz dieser Dimension a priori vorzusetzen. Zum anderen sollte das intersektionale Potential des Habituskonzepts und der Habitushermeneutik ausgelotet werden: Für jedes Interview wurde eine habitushermeneutische Interpretation vorgenommen und dabei versucht, zugleich die Dimensionen von Klasse und Geschlecht

\footnotetext{
${ }^{11}$ Auch in der Ansprache der Studierenden in den Seminaren wurde ,Geschlecht ${ }^{\text { nicht }}$ explizit thematisiert, sondern, wie bereits dargestellt, auf die übergeordnete Forschungsfrage verwiesen, wie Studien(fach)wahlen zustande kommen. Testweise wurde in einigen ausgewählten Interviews nach der Bedeutung von Geschlecht gefragt (etwa: ,Wie ist der Anteil von Frauen und Männern in einem Studiengang und was denkst du darüber ${ }_{i}$ ), was aber nur wenig erkenntnisreich schien. Bei der Interpretation dieser Passagen wird berücksichtigt, dass Geschlecht durch die Interviewerin thematisiert wurde.
} 
im Habitus in die Interpretation einzubeziehen. Es wurden also keine getrennten Interpretationsverfahren für Klasse und Geschlecht angesetzt, sondern der gleichzeitige Blick auf beides und somit die entsprechende Verwobenheit gelegt. Im gleichen Zug wurde versucht, von der müßigen Frage der Dominanz einer der beiden Dimensionen Abstand zu nehmen ${ }^{12}$.

\section{Die Erfassung des „fraglos Gegebenen“}

Wenn also Geschlecht in der Interviewführung nicht zur Leitdifferenz erhoben und seine Bedeutung für die Befragten aktiv abgefragt wird, stellt sich die Frage, wie es in der Analyse überhaupt berücksichtigt werden kann. Dies verweist auf das Problem der qualitativen Geschlechterforschung, wie das „fraglos Gegebene“ analysiert werden kann, wie sich also „... ein Gegenstand empirisch fassen [lässt], der den Befragten als Selbstverständlichkeit gegeben ist?“ (Behnke/Meuser 1999: $51 \mathrm{f}$.). Und auch hier knüpft die gewählte Auswertungsmethode an: So ist der Habitus allgemein, wie seine Dimension von Geschlecht im Speziellen, ebenso „fraglos gegeben“ und gekennzeichnet durch seinen hohen vorreflexiven Anteil. Die Habitushermeneutik hat sich in den bereits genannten Studien mehrfach als fähig erwiesen, diese fraglos gegebenen Muster des Habitus zu entschlüsseln. Die gleiche Hoffnung kann also in das Potential der Methode gesetzt werden, der Geschlechtsdimension des Habitus nachzuspüren und so unhinterfragte Prozesse der Vergeschlechtlichung offenzulegen.

\subsubsection{Der habitushermeneutische Blick auf Geschlecht}

Nachdem deutlich geworden ist, welche Aspekte im empirischen Umgang mit Geschlecht berücksichtigt werden sollten, wird nun umrissen, wie dies in der Analyse umgesetzt wurde. Wie lässt sich die Habitusdimension von Geschlecht bei der Anwendung der Habitushermeneutik greifen?

Auch dabei wurde an die methodologische Grundannahme angeknüpft, dass sich Akteur*innen ständig ,implizit zuordnen und abgrenzen“( (ebd.), was mit

\footnotetext{
${ }^{12}$ Was sich je nach Zusammensetzung der Interpretationsgruppen als nicht einfach erwies: So liefen die Interpretationsprozesse in stärker von Geschlechterforscher*innen besetzten Gruppen Gefahr, den analytischen Blick besonders auf ,Geschlecht" scharf zu stellen, während bei Bildungsforscher*innen tendenziell die soziale Herkunft mehr Raum in den Analysen einnahmen. Dass das Material in ebendiesen unterschiedlichen Gruppen analysiert wurde und die beiden ,scharf gestellten " Dimensionen in der weiteren Fallanalyse verknüpft wurden, eröffnet das Potenzial, keine der beiden Dimensionen verkürzt zu behandeln.
} 
den gesellschaftlichen Teilungen - also auch mit der geschlechtlichen Teilung zusammenhängt. Für die habitushermeneutische Analyse von Geschlecht bedeutet das, implizite vergeschlechtlichte Zuordnungen, Selbstpositionierungen, Zuschreibungen und Abgrenzungen in den Fokus zu nehmen. Dabei bietet es sich an, auf der Grundlage der Erkenntnisse der Geschlechterforschung zur Berufs- und Studien(fach)wahl (s. Abschnitt 3.1) bestimmte Passagen im Interview mit dem Fokus auf Geschlecht zu betrachten und an diese Passagen im Prozess der habitushermeneutischen Analyse spezifische Fragen zustellen wie etwa:

- Über welche Personen erzählen die Befragten, wenn es um ihre berufliche Orientierung geht? Wie werden diese Personen beschrieben?

- Welche (impliziten) geschlechtlichen Zuschreibungen nehmen die Befragten vor? Welche Selbstpositionierungen werden dabei deutlich?

- Wie ist die Arbeitsteilung in der Herkunftsfamilie der Befragten organisiert? Wie positionieren sich die Befragten hierzu? Antizipieren sie die Gründung einer eigenen Familie und welche Arbeitsteilung verknüpfen sie damit?

Indem diese Fragen an den entsprechenden Stellen im Material verfolgt werden, lässt sich entschlüsseln, welche Vorstellungen von Weiblichkeit und Männlichkeit sich in den Habitus der Befragten eingeschrieben haben. Zudem werden Hinweise erkennbar, wie sich diese Vorstellungen in der Praxis der Befragten niederschlagen. So ergibt sich ein Einblick in verschiedene Dimensionen der Vergeschlechtlichung des Habitus und deren Bedeutung für die Studien(fach)wahl.

Zentral bei diesem interpretativen Vorgehen ist die ständige Vergegenwärtigung, dass ebendiese Geschlechtsdimension des Habitus stets eine klassenspezifische ist (s. Kapitel 4). Wie die Befragten ihren Weg in das Studium gehen, welchen Beruf sie ergreifen wollen und welche Zukunft sie antizipieren ist ein Ausdruck ihrer sozialen Position als Frau bzw. Mann eines bestimmten Milieus. Durch dieses Zusammendenken von Geschlecht und Klasse in der habitushermeneutischen Analyse soll das Zusammenspiel der beiden Ungleichheitsdimensionen in der Entstehung von Studien(fach)wahlen nachgezeichnet werden. 
Open Access Dieses Kapitel wird unter der Creative Commons Namensnennung 4.0 International Lizenz (http://creativecommons.org/licenses/by/4.0/deed.de) veröffentlicht, welche die Nutzung, Vervielfältigung, Bearbeitung, Verbreitung und Wiedergabe in jeglichem Medium und Format erlaubt, sofern Sie den/die ursprünglichen Autor(en) und die Quelle ordnungsgemäß nennen, einen Link zur Creative Commons Lizenz beifügen und angeben, ob Änderungen vorgenommen wurden.

Die in diesem Kapitel enthaltenen Bilder und sonstiges Drittmaterial unterliegen ebenfalls der genannten Creative Commons Lizenz, sofern sich aus der Abbildungslegende nichts anderes ergibt. Sofern das betreffende Material nicht unter der genannten Creative Commons Lizenz steht und die betreffende Handlung nicht nach gesetzlichen Vorschriften erlaubt ist, ist für die oben aufgeführten Weiterverwendungen des Materials die Einwilligung des jeweiligen Rechteinhabers einzuholen.

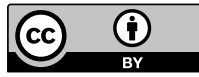

